\title{
Matrix-assisted laser desorption/ionisation-time of flight mass spectrometry (MALDI-TOF MS) in clinical mircobiological routine diagnostics ${ }^{1)}$
}

\author{
Sören Schubert* and Andreas Wieser \\ Max von Pettenkofer Institute for Hygiene and Medical \\ Microbiology, Munich, Germany
}

\begin{abstract}
Very recently a novel method for differentiation of bacteria and fungi was developed, that is, identification by means of matrix-assisted laser desorption/ionisation-time of flight mass spectrometry (MALDI-TOF MS). This differentiation relies on the exact measurement of species-specific protein spectra of ribosomal proteins. It is at least as accurate as conventional biochemical differentiation methods, but provides results within minutes. In addition to differentiation of bacteria and yeasts grown on agar plates, direct identification is feasible from positive blood cultures as well as from urine samples of patients suffering from urinary tract infections. Future developments of MALDI-TOF MS for clinical microbiological purposes include the detection of $\beta$-lactamase and carbapenemase activity as well as genotyping of bacteria below the species level.
\end{abstract}

Keywords: bacterial species; blood culture; differentiation; matrix-assisted laser desorption/ionisation-time of flight (MALDI-TOF); yeast.

\section{Content and methodology of microbiological diagnostics}

The first objective of clinical microbiological diagnostics is, in patient samples, to identify the pathogens (bacteria, fungi, parasites) causing a disease and - in the case of bacteria and

\footnotetext{
${ }^{1)}$ Original German online version at: http://www.reference-global. com/toc/labm/35/4. The German article was translated by Compuscript Ltd. and authorized by the authors.

*Correspondence: PD Dr. med. Sören Schubert, Max von

Pettenkofer Institute for Hygiene and Medical Microbiology, Ludwig-Maximilians-University Munich, Marchioninistr. 17, 81377 Munich, Germany

Tel.: +49892180 78202

Fax: +4989218078294

E-Mail: schubert@med.uni-muenchen.de
}

yeast-like fungi - to determine resistance testing for antimicrobial agents (antibiogram). The unequivocal species identification of pathogens from clinical materials is a central task. The classical microbiological diagnosis is based on microscopy of specimens and the cultivation on nutrient media. For more than five decades, primarily biochemical assays have been used to differentiate bacteria and fungi, in which the sum of the genus and/or species-specific metabolic properties are put together to form a usable diagnostic biochemical reaction profile. Major drawbacks of this biochemical differentiation principle are the long incubation periods for biochemical reactions (6-12 h) and the variability of individual metabolic characteristics as well as significant ongoing cost of the components of the tests. Furthermore, increased demands are placed on the expertise and experience of the performing laboratory personnel, as a predifferentiation of bacteria (e.g., Gram stain, Kligler agar and catalase or oxidase reaction) may be necessary under certain circumstances. In recent years a completely new approach for the differentiation of bacteria and fungi has moved from the development stage to being a routine method - the identification of pathogens by MALDI-TOF MS, or matrix-assisted-laserdesorption/ionisation-time of flight mass spectrometry. This mass spectrometric method has, in principle, the potential to replace the classical biochemical differentiation methods in microbiological diagnostics, because it combines significant advantages such as very short analysis times, ease of use and automation with low consumable costs, as well as high sensitivity and specificity [1].

\section{MALDI-TOF MS-methodological foundations}

MALDI-TOF MS has been used for several years with success in proteome research to determine the mass of proteins and peptides and to identify in combination with 1-D and 2-D gel electrophoresis unknown proteins [2] (Figure 1). The technology is used for mass analysis of chemical compounds and is a combination of matrix-assisted laser-desorption/ionisation (MALDI) and time of flight (TOF).

In the field of clinical chemistry MALDI-TOF MS has become an established method for the detection of specific biomarkers in the diagnosis of tumour diseases, Alzheimer's disease, rheumatoid arthritis and allergies [2]. In addition to 


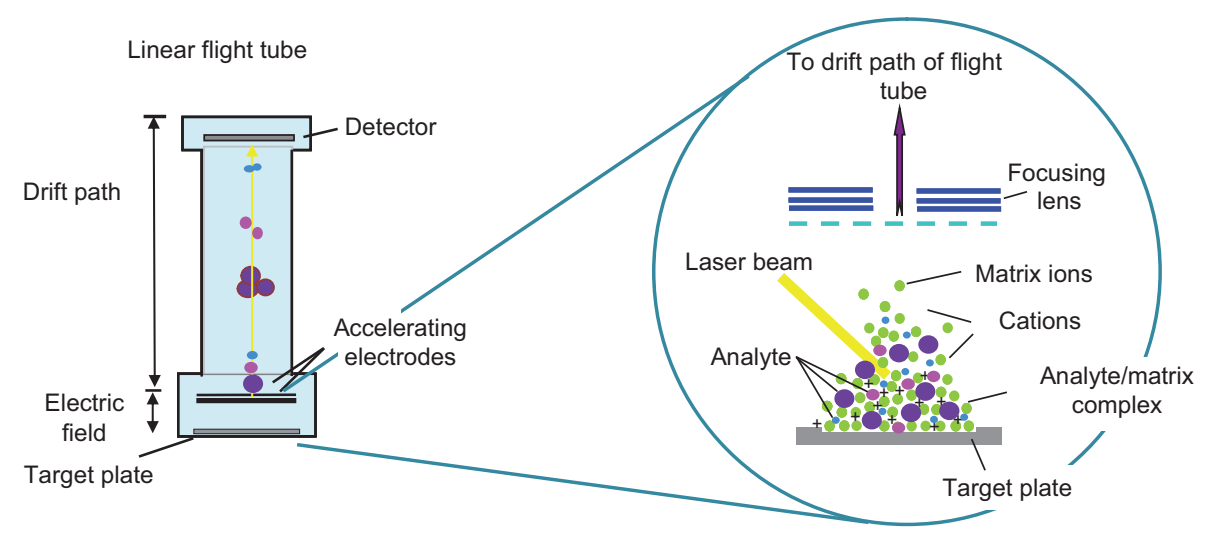

Figure 1 Principle of MALDI-TOF MS-based identification of proteins and peptides.

The sample and/or the bacterial or fungal culture with the proteins therein (analytes, shown as blue, pink and purple spheres) are applied to the target plate together with the matrix (green spheres). After firing the laser, the desorbed proteins are accelerated in an electric field. Then, the analytes are separated in the drift path by charge and mass. By determining the time of flight of each analyte, characteristic mass spectra are created.

proteins and peptides, MALDI-TOF MS can also be used to detect nucleic acids.

Initial experiments to use the mass spectrometric methods for the identification of microorganisms were performed in 1975 [3]. The development of MALDI-TOF MS in the 1980s marked the first time relatively large biomolecules could be analysed [4]. Subsequently, work was started to use MALDITOF MS to identify different species of bacteria $[1,5,6]$. These initial studies were characterised by poor reproducibility of results, and by the dependence of test results on the growth conditions and the different culture media. Routine use of this method only became possible when the studies of low molecular mass ranges $(<1000 \mathrm{Da})$ had been moved to a mass range between 2000 and 12,000 Da. By refining the methodology and the software-based analysis, MALDI-TOF MS can now be used also in the routine laboratory to differentiate microorganisms.

Since the MALDI-TOF mass spectrometry is a very sensitive method, only a small amount of the pathogen (bacteria, ranging from $10^{4}$ to $10^{6} \mathrm{CFU}$ ) has to be applied to the MALDI sample plate (target) for analysis (Figure 2). To embed this sample, for example, a $1 \mu \mathrm{L}$ matrix solution is applied and dried. In the process, the matrix (cinnamic or benzoic acid derivates) co-crystallises with the pathogen on the steel surface of the target plate, which carries 12-160 assay areas. The loaded target plates are introduced into the measuring chamber of the mass spectrometer, which is under continuous high vacuum conditions. In order to maintain these conditions, vacuum pumps are in use, which pump out the air introduced during the loading process. In the evacuated measuring chamber, the individual assay areas are addressed and parts of the sample are vaporised explosively through a number of short laser pulses together with the matrix. This causes an ionisation of the pathogen proteins that are accelerated as analytes in the electric field of a high voltage source (10-30 $\mathrm{kV}$ ). The time of flight to impact of the analytes on the detector is measured accurately in the high vacuum conditions.
Both the degree of ionisation and the mass of the proteins determine the time of flight of the proteins and produce a characteristic spectrum for each pathogen, which is a kind of mass spectrometric fingerprint. For identification purposes, it is mainly the mass spectrum of positively charged ribosomal proteins between 2 and $12 \mathrm{kDa}$ that is used, which provides very stable and meaningful spectra (Figure 3). These are hardly influenced by the growth conditions of the microorganisms.

The spectra measured by the instrument are adjusted and synchronised fully automatically with reference databases, which contain a large number of spectra of medically relevant isolates. The database synchronisation can be automated by new algorithms and take place in near real-time [7, 8]. This yields a very short turnover time until a positive result is obtained. If only one sample is to be measured, it is possible to obtain, from the application of the sample and within 5-7 min, a species identification. If a fully-loaded target plate with 96 isolates is to be loaded with samples and measured, times of less than an hour from the application of the first sample to the measurement of the last isolate are possible. The software comparison of the measured spectrum with the spectra stored in the database can, of course, never produce perfectly identical measured values. Therefore, the software generates a numerical value (score-value), which indicates the validity of the differentiation result (Figure 4). Furthermore, the software gives the best result (best match) and even more results in order to facilitate the plausibility check (Figure 4). The measured results can be fed directly into the laboratory computer system via a computer connection.

\section{Use of MALDI-TOF mass spectrometry in microbiological diagnostics - pathogen differentiation of culture media}

Studies comparing MALDI-TOF MS-based and conventional, biochemical differentiation of bacteria and yeast-like 


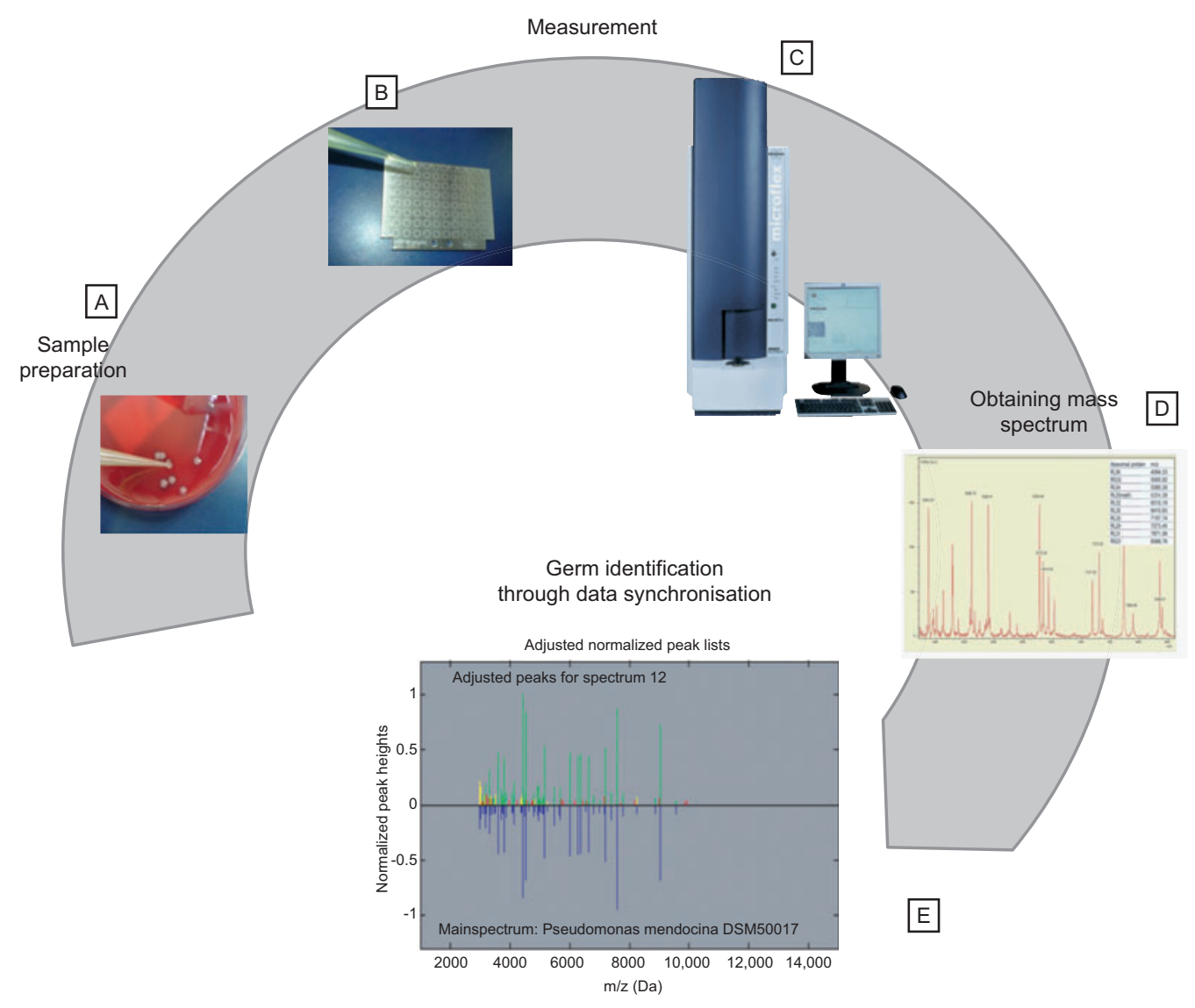

Figure 2 Workflow.

(A) Subculturing of bacterial colonies from the agar plate. (B) Application of a bacterial colony and matrix on the target. (C) Measurement in MALDI-TOF. (D) Measurement result as a spectrum. (E) Germ identification by automatic synchronisation of the spectrum with the database.

fungi have documented a high validity of the MALDI-TOF results. A retrospective study [9] of 1116 isolates of bacteria mainly encountered in a clinical microbiological laboratory setting showed a correct identification by MALDI-TOF MS in $95.2 \%$ of cases. In another recently published prospective study it has been shown [10] that of 1660 bacterial isolates from 109 different species, $84.1 \%$ were identified correctly at species level by MALDI-TOF MS. At this point, still missing database entries in several studies have been blamed for unsuccessful identification.

Own validation studies of the MALDI-TOF MS system (Bruker Daltonik) in comparison with biochemical differentiation systems (BD Phoenix ${ }^{\mathrm{TM}}, \mathrm{API}^{\circledR}$-bioMérieux) and any subsequent sequencing of 16S-rDNA/28S-rDNA have yielded comparable results. In 1200 prospectively studied isolates, including 370 Enterobacteriaceae, 300 non-fermenters, 110 other Gram-negative bacteria, 320 Gram-positive isolates and 100 yeast-like fungi, a concordant result was achieved between MALDI-TOF MS and biochemical differentiation in $93.5 \%$ of cases. In $3 \%$ of the cases, biochemical differentiation was superior, in $3.5 \%$ of the cases, the MALDI-TOF MS method provided a more accurate result when the 16S rDNA sequencing, which was also carried out, was considered a gold standard. In many cases where the MALDI-TOF MS analysis yielded poorer results, only the relevant reference spectra of the isolates had not been deposited in the database yet. Meanwhile, by creating new reference spectra and corresponding extensions of commercial databases, it was possible largely to close the diagnostic gap. Unlike biochemical test systems, the MALDI-TOF MS system is an open system, in which the database can also be extended by the user independently. Through appropriate additions to the reference spectra of the databases, it was possible to achieve a nearly $100 \%$ identification rate of Neisseria [11], clostridia [12], mycobacteria [13], salmonella [14], viridans streptococci [15] and Helicobacter pylori [16]. While the MALDI-TOF MS system provides comparably good results in comparison to biochemical test systems in the differentiation of enterobacteria, staphylococci and streptococci, it shows clear advantages in the differentiation of Gram-positive rods [17], anaerobes and some non-fermenters $[18,19]$. Particularly with those pathogens that are characterised by long culture times, MALDI-TOFbased diagnostics has yielded clear time advantages, which have drastically reduced the time to obtain differentiation results in the lab. 


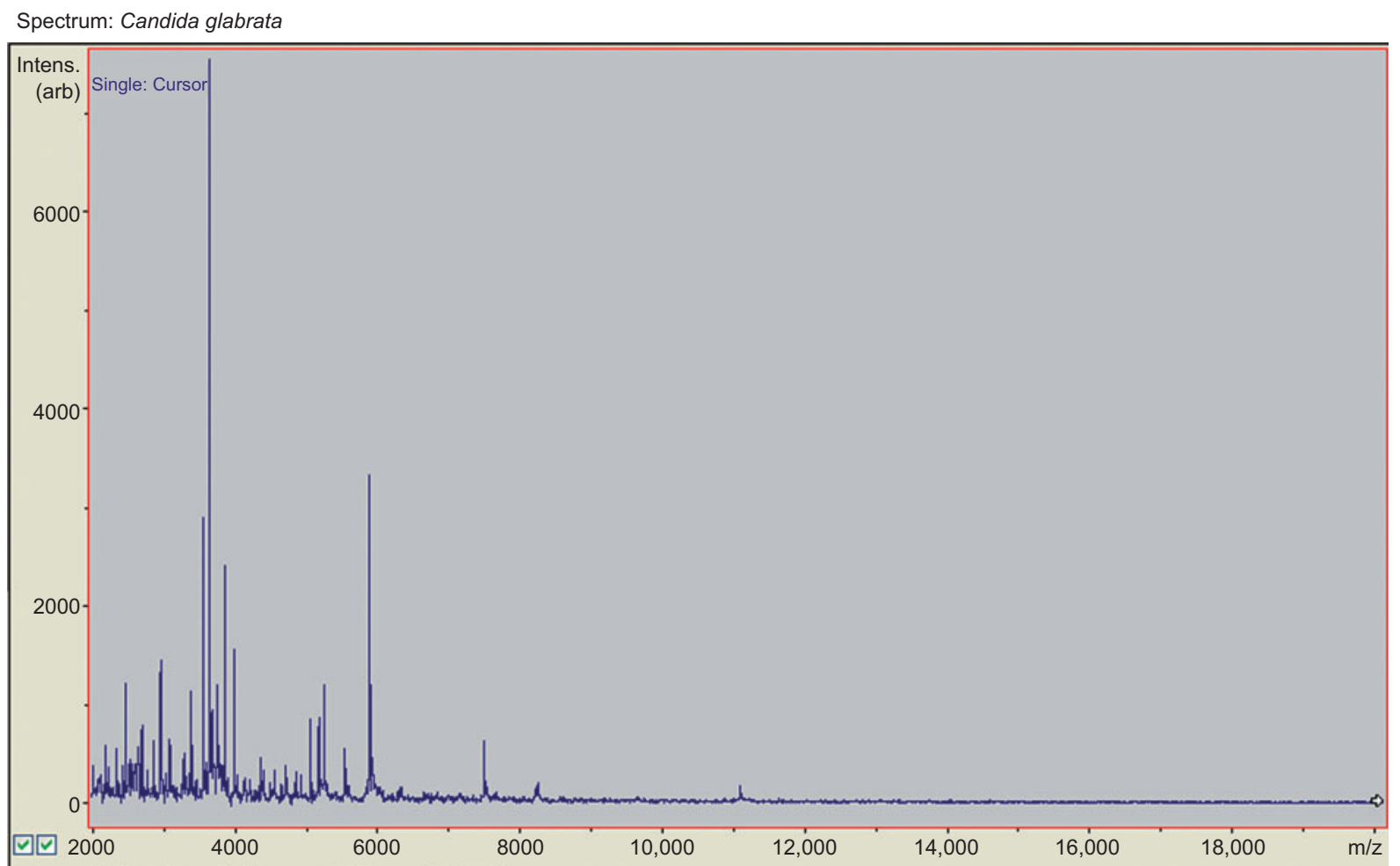

Figure 3 Mass spectrum of a Candida glabrata sample.

In the measured range 4000-8000 Da, characteristic mass peaks can be seen, which represent predominantly ribosomal proteins. The pattern of such a spectrum is compared with the appropriate database entries by means of MALDI evaluation software.

MALDI-TOF MS has already been used successfully by various groups to differentiate yeast-like fungi and moulds. It was shown that $96 \%$ of 250 clinical Candida isolates from 15 species could be identified correctly by means of
MALDI-TOF MS [20]. In two prospective studies to identify yeast-like fungi, the rates of correct differentiation results were similarly high [21, 22]. Far fewer data are available for the differentiation of moulds such as Aspergillus, Penicillium

Result after database synchronisation

\begin{tabular}{|c|c|c|c|}
\hline $\begin{array}{c}\text { Rang } \\
\text { (Qualität) }\end{array}$ & Übereinstimmende Muster & $\begin{array}{c}\text { Bewertungs- } \\
\text { zahl }\end{array}$ & $\begin{array}{l}\mathrm{NCBI} \\
\text { code }\end{array}$ \\
\hline $\begin{array}{c}1 \\
(++)\end{array}$ & Candida glabrata ATCC 90030 VML & 2.187 & $\underline{5478}$ \\
\hline $\begin{array}{c}2 \\
(++)\end{array}$ & Candida glabrata DSM 70615 DSM & 2.132 & $\underline{5478}$ \\
\hline $\begin{array}{c}3 \\
(++)\end{array}$ & Candida glabrata ATCC 2001T THL & 2.038 & $\underline{5478}$ \\
\hline $\begin{array}{c}4 \\
(++)\end{array}$ & Candida glabrata DSM 11950 DSM & 2.01 & $\underline{5478}$ \\
\hline $\begin{array}{c}5 \\
(+)\end{array}$ & Candida glabrata DSM 6425 DSM & 1.974 & $\underline{5478}$ \\
\hline $\begin{array}{c}6 \\
(-)\end{array}$ & Arthrobacter citreus IMET 10680T HKJ & 1.257 & 1670 \\
\hline $\begin{array}{c}7 \\
(-)\end{array}$ & Lactobacillus agilis DSM 20508 DSM & 1.227 & $\underline{1601}$ \\
\hline $\begin{array}{c}8 \\
(-)\end{array}$ & Clostridium novyi A 1025_NCTC 538 BOG & 1.225 & 1542 \\
\hline $\begin{array}{c}9 \\
(-)\end{array}$ & Pseudomonas rhizosphaerae LMG 21640T HAM & 1.2 & $\underline{216142}$ \\
\hline $\begin{array}{l}10 \\
(-)\end{array}$ & Agromyces fucosus HKI 11529_DSM 8597T HKJ & 1.195 & $\underline{41985}$ \\
\hline
\end{tabular}

Figure 4 The results of the database comparison can be displayed in tabular form and sent to the laboratory computer automatically. Here the results are assigned a numerical value for the validity of differentiation ("score") in order to document the validity of the identification result. 
and Fusarium or dermatophytes [23-27]. The different conditions of moulds with the formation of mycelia and conidia and correspondingly different protein patterns within an isolate complicate MALDI-TOF MS identification [28]. Therefore, some adjustments to the measurement and evaluation must be made before the MALDI-TOF measurement of mould is fully suitable for routine operations. Since MALDI-TOF differentiation is based on cultivated fungi and the cultivation of dermatophytes is very time consuming, it remains to be seen whether MALDI-TOF diagnostics can prevail in this area. Many routine laboratories do not offer a diagnosis of dermatophytes, especially as the clinical relevance of accurate identification is low in many cases (e.g., athlete's foot).

Unlike in the early stages of mass spectrometric differentiation of pathogens, today's MALDI-TOF MS-based examination is largely independent of the nature of the culture medium. As a result, apart from standard culture media like blood and chocolate agar, selective culture media like McConkey agar or XLD agar can also be used for differentiation [6]. As the identification of isolates requires very little culture material, sometimes colonies of (sub-)cultures that arise after a few hours of incubation will do. In this way, mixed cultures can be converted to pure cultures and may be identified on the same day. The investigated colonies, however, should be as fresh as possible (not more than $48 \mathrm{~h}$ ), because with increasing age of the colonies, the ribosomal proteins of the bacteria become degraded. As a result, MALDI-TOF MS spectra tend to be weaker and less differentiated, which can lead to difficulty in data comparison and to poorer differentiation results.

In most cases, MALDI-TOF MS differentiation of bacteria can be carried out without sample preparation by simply spreading the bacteria colony on the target plate. Some pathogens that have a stable cell wall (e.g., yeast-like fungi) may require a short extraction step to render the ribosomal proteins available for analysis. For this purpose, for example, the colony to be tested is placed in $80 \%$ ethanol, centrifuged and then resuspended in formic acid and acetonitrile. The resulting supernatant is applied to the target plates after centrifugation. Through this extraction step, it is usually possible to generate spectra that are clearer and more complex by multiple factors, which then allow for a successful database search. After cleaning and disinfection with $70 \%$ ethanol, the target plate can immediately be used again for further measurements.

Since the MALDI-TOF MS analysis so far does not allow for routine antibiotic or antimycotic resistance testing of isolates, conventional manual, semi-automatic or automatic procedures will continue to be necessary over the medium term. The MALDI-TOF MS system used for differentiation, however, can easily be combined in routine operations with manual and automatic resistance testing methods. An integration of the MALDI-TOF MS analysis with the laboratory computer system using uni- and bi-directional communications, however, is already feasible and has been implemented in some larger diagnostic centres in their routine operations. Thus, the differentiation results can be sent online to the machine for resistance testing and be used by it to interpret the resistance, or resistance mechanisms.

\section{Use of MALDI-TOF mass spectrometry in microbiological diagnostics - direct identification of pathogens from positive blood cultures}

Early on, attempts were made to utilise MALDI-TOF MS for the differentiation of bacteria and fungi directly from positive blood cultures [29]. The problem with this application is that large amounts of proteins in the patient's blood cells obscure the detection of specific bacteria and fungal proteins. Several research groups have developed as a result different sample preparation protocols, which separate bacteria from the host proteins either by way of differential centrifugation or cell lysis methods [30-32]. The studies on direct detection show proper identification rates at the species level of $75 \%-95 \%[33,34]$. Many of the centrifugation methods are either time consuming, and difficult to reconcile with the routine workflow of diagnostic laboratories, or not efficient enough. Lysis procedures are currently favoured. With this fast purification process, it is possible to achieve correct identifications in $86 \%$ of cases within 30 min. Apart from the difficulties of delimitation of Streptococcus pneumoniae and Streptococcus mitis/oralis, no false results have been documented in connection with direct blood cultures. Many studies require an adaptation of the MALDI-TOF score thresholds for the validation of the results of blood culture differentiation. The reason is that even measurements with lower score values that are assessed for the measurement of bacterial colonies as being not sufficiently valid do yield correct results in connection with direct blood culture identification [32].

\section{Use of MALDI-TOF mass spectrometry in microbiological diagnostics - pathogen detection directly from patient samples (urine samples)}

The quick and easy identification of pathogens by means of MALDI-TOF from the culture can only partially be transferred directly to patient samples. This is first and foremost due to the method itself, which detects pathogen proteins and produces characteristic spectra. Samples obtained directly from a patient, such as sputum, blood or cerebrospinal fluid, however, contain much of the patient's protein and only relatively little of the pathogen. Thus, it is hardly possible to separate the individual spectra and filter out those of pathogens. In addition, in many materials one encounters a more or less complex normal flora, whose signals must be separated from the pathogens. In materials naturally low in protein, with high pathogen load and lack of normal flora such as urine, it is still possible to carry out direct analyses successfully [35]. For this purpose, the samples are first centrifuged slowly to pellet leukocytes. The second step then 
involves a quick centrifugation to separate the pathogens. After another washing step with distilled water, the pathogens are purified sufficiently to be analysed successfully by means of MALDI-TOF MS. Since urinary tract infection is caused in most cases only by a predominant pathogen, only few problems arise with mixed spectra. With such protocols, larger studies achieved correct identification rates of $91.8 \%$ to the species level directly from urine (bacterial load significantly $>10^{5} \mathrm{CFU} / \mathrm{mL}$ ) [35]. Thus, the diagnosis by means of MALDI-TOF is a real alternative to lengthier and more expensive culture-based methods, especially if done on an outpatient basis with no resistance testing.

\section{Use of MALDI-TOF mass spectrometry in microbiological diagnostics - advantages, opportunities and limitations}

The MALDI-TOF MS-based pathogen identification as stated above is based on the analysis of mostly ribosomal proteins. Therefore, the differentiation patterns resemble those of the 16S-rDNA sequence comparisons. As a result of this, the MALDI-TOF MS method is not able to separate microorganisms that are genetically very closely related. This is clinically significant, for example, in Shigella sp. and Escherichia coli or S. pneumoniae (pneumococci) and representatives of the $S$. oralis/mitis group. In these cases, the use of biochemical supplementary tests, antigen-detection or molecular methods continues to be necessary. On the other hand, the MALDI-TOF MS analysis makes apparent the shortcomings of conventional species differentiation based on metabolic reactions, whose selectivity may be limited by the loss or gain of metabolic properties. This is the reason why the comparison between biochemical and molecular and/ or MALDI-TOF MS-based differentiation produces in a few cases discordant results based on different methodological approaches. The enormous gain in time, the high accuracy and easy automation of the MALDI-TOF-MS analysis are the main advantages of this method in routine laboratory work. A single system for identifying the various groups of bacteria and yeast-like fungi can be used; a preliminary examination of the isolates is not required. Since the differentiation results are available after just a few minutes and on the day of the first material reading, that is, $12-24 \mathrm{~h}$ after receipt of the sample, valuable interim or preliminary reports can be shared much earlier with the other doctors treating the patient.

Another advantage of the MALDI-TOF MS method is that the reference spectra of new isolates can be integrated into the system by the user both as regularly commercially available database updates and also internally at the lab. A feasible option may also include a "public domain" where MALDI-TOF users can store spectra. Accordingly, pathogen groups or species not sufficiently represented in the database so far could be added continuously. One must only ensure that only valid audited spectra can actually be entered in the database, since otherwise the identification quality may suffer.

The disadvantage of the MALDI-TOF MS analysis has been that the direct detection of pathogens from patient samples is very difficult and therefore almost always requires a preculture. Although there are promising initial MALDI-TOF MS studies to determine bacterial resistance to $\beta$-lactam antibiotics and carbapenems, so far MALDITOF MS studies have not allowed for significant observations on the sensitivity of isolates to anti-infectives in routine operations. Nor should it be expected that in future MALDI-TOF MS can be used to obtain a complete antibiotic resistance profile of bacterial isolates with respect to different substance classes. The mechanisms are too different and the expression of individual resistance factors is too low for that.

The cost of MALDI-TOF MS analysis equipment suitable for routine diagnostics, including computers and software with an integrated database, currently comes to $€ 180,000$ to $€ 200,000$ and is still relatively high. By contrast, the operating costs of $<50$ cents per analysis are very small. In addition, a high sample throughput can be achieved with only one device. In the near future it is expected that improvements in semiconductor laser technology will lead to longer serviceable life and also speed up the possible pulse bursts. This would speed up the operation further and reduce the running costs.

The introduction of automatic cleaning systems of the ion source ("source heating") can lengthen the intervals for routine cleaning and thus help to reduce maintenance costs and maintenance-related failures. In one study, the cost of MALDI-TOF MS identification was calculated to a quarter of the corresponding biochemical studies [10]. Here, the authors have included consumables, salaries of employees and equipment depreciation over a 5 -year period. Similar cost reductions are reported in another study [36]. No doubt, the issue of cost reduction must be examined separately for each laboratory environment; statements derived from studies are not always fully transferable.

\section{Future applications of MALDI-TOF mass spectrometry in microbiological diagnostics}

Currently further MALDI-TOF MS applications are being developed for clinical microbiological diagnostics. On the one hand, it is attempted to use the technology for the determination of selected antibiotic resistances, such as the detection of penicillin-binding protein PBP2a in MRSA [37] or defined $\beta$-lactamase types in Enterobacteriaceae. On the other hand, development work is focused on the direct detection of pathogens from patient samples. There are also studies to see whether the MALDI-TOF MS method may be used in future for genotype analysis to clarify epidemiological issues. In a way similar to the known restriction fragment length polymorphism (RFLP) typing, mass spectrum typings are used that are similarly characteristic of strains.

MALDI-TOF MS has already been used to differentiate between methicillin-resistant Staphylococcus aureus isolates (MRSA) and methicillin-sensitive (MSSA) ones [38, 39]. This did not involve detecting the MRSA typical penicillinbinding protein PBP2a, but rather examined the patterns of 
cell wall proteins. Thus, the clonality of $S$. aureus isolates can be shown, but not all isolates of a "typical" MRSA clone are actually also MRSA (e.g., loss of PBP2a). It is not surprising therefore that even conflicting data have been published $[40,41]$.

A promising approach for the identification of resistance to $\beta$-lactam antibiotics is the MALDI-TOF MS-based detection of cleavage products of $\beta$-lactam antibiotics and carbapenems from the influence of corresponding bacterial enzymes, $\beta$-lactamases and carbapenemases. Through the influence of these enzymes, the $\beta$-lactam or penem ring in the molecule of the respective antibiotics is cleaved by hydrolysis. This corresponds to the addition of an $\mathrm{H}_{2} \mathrm{O}$ molecule and subsequent decarboxylation and/or addition of Na. After 1-3 h of incubation of the antibiotic with a suspension of the test strain, MALDI-TOF MS can be used to show the change in mass of the antibiotic molecule present and thus to represent the effect of bacterial enzymes in the test antibiotic. In this way, resistance to various penicillins, cephalosporins and carbapenems, such as meropenem and imipenem, can already be demonstrated (ECCMID 2011, Poster, Sparbier et al.).

Further developments are moving to MALDI-TOF MS being used to type bacterial isolates below the species level in order to make epidemiological observations on individual patient or environmental isolates. The objective in this is to carry out individual strain typing by way of discrete differences in the spectra obtained, and thus to render superfluous time-consuming and costly molecular tests (PCR, PFGE).

Of great interest, particularly for laboratories with a high sample throughput, is the development of processing lines. In addition to creating agar plates and liquid media, incubation processes and digital photography of the overgrown agar plates for diagnosis are components of this new strategy. Meanwhile, several companies have integrated MALDI-TOF MS into this concept, where robots pick up colonies from agar plates and apply them to MALDI target plates. But this development is still in its infancy.

\section{Conclusions}

The currently commercially available diagnostic MALDITOF MS solutions enable automated germ differentiation of cultured bacteria and fungal species. Overall, MALDITOF MS-based procedures can be counted on to produce significant changes and improvements in diagnostic microbiology. The clinical evaluation of the data collected by an experienced specialist in microbiology, virology and epidemiology of infectious diseases gains a high priority in this process.

\section{Acknowledgments}

Our work on the further development of diagnostic MALDI-TOF MS technology is supported by Bayerische Forschungstiftung, the Bavarian Research Foundation, (Research Network FORPROTECT - protection from infectious diseases through new diagnostic techniques and treatment approaches).

\section{References}

1. Claydon MA, Davey SN, Edwards-Jones V, Gordon DB. The rapid identification of intact microorganisms using mass spectrometry. Nat Biotechnol 1996;14:1584-6.

2. Marvin LF, Roberts MA, Fay LB. Matrix-assisted laser desorption/ionization time-of-flight mass spectrometry in clinical chemistry. Clin Chim Acta 2003;337:11-21.

3. Anhalt JP, Fenselau C. Identification of bacteria using mass spectrometry. Anal Chem 1975;47:219-25.

4. Hillenkamp F, Karas M. Mass spectrometry of peptides and proteins by matrix assisted ultraviolet laser desorption/ionization. Methods Enzymol 1990;193:280-95.

5. Demirev PA, Ho YP, Ryzhov V, Fenselau C. Microorganism identification by mass spectrometry and protein database searches. Anal Chem 1999;71:2732-8.

6. Fenselau C, Demirev PA. Characterization of intact microorganisms by MALDI mass spectrometry. Mass Spectrom Rev 2001;20:157-71.

7. Jarman KH, Cebula ST, Saenz AJ, Petersen CE, Valentine NB, Kingsley MT, et al. An algorithm for automated bacterial identification using matrix-assisted laser desorption/ionization mass spectrometry. Anal Chem 2000;72:1217-23.

8. Sauer S, Freiwald A, Maier T, Kube M, Reinhardt R, Kostrzewa $\mathrm{M}$, et al. Classification and identification of bacteria by mass spectrometry and computational analysis. PLoS ONE 2008;3:e2843.

9. Eigner U, Holfelder M, Oberdorfer K, Betz-Wild U, Bertsch D, Fahr AM. Performance of a matrix-assisted laser desorption ionization-time-of-flight mass spectrometry system for the identification of bacterial isolates in the clinical routine laboratory. Clin Lab 2009;55:289-96.

10. Seng P, Drancourt M, Gouriet F, La SB, Fournier PE, Rolain JM, et al. Ongoing revolution in bacteriology: routine identification of bacteria by matrix-assisted laser desorption ionization timeof-flight mass spectrometry. Clin Infect Dis 2009;49:543-51.

11. Ilina EN, Borovskaya AD, Malakhova MM, Vereshchagin VA, Kubanova AA, Kruglov AN, et al. Direct bacterial profiling by matrix-assisted laser desorption - Ionization time-of-flight mass spectrometry for identification of pathogenic neisseria. J Mol Diagn 2009;11:75-86.

12. Grosse-Herrenthey A, Maier T, Gessler F, Schaumann R, Bohnel H, Kostrzewa M, et al. Challenging the problem of clostridial identification with matrix-assisted laser desorption and ionization-time-of-flight mass spectrometry (MALDI-TOF MS). Anaerobe 2008;14:242-9.

13. Pignone M, Greth KM, Cooper J, Emerson D, Tang J. Identification of mycobacteria by matrix-assisted laser desorption ionization-time-of-flight mass spectrometry. J Clin Microbiol 2006;44:1963-70.

14. Dieckmann R, Helmuth R, Erhard M, Malorny B. Rapid classification and identification of salmonellae at the species and subspecies levels by whole-cell matrix-assisted laser desorption ionization-time of flight mass spectrometry. Appl Environ Microbiol 2008;74:7767-78.

15. Friedrichs C, Rodloff AC, Chhatwal GS, Schellenberger W, Eschrich K. Rapid identification of viridans streptococci by mass spectrometric discrimination. J Clin Microbiol 2007;45:2392-7.

16. Ilina EN, Borovskaya AD, Serebryakova MV, Chelysheva VV, Momynaliev KT, Maier T, et al. Application of matrix-assisted laser desorption/ionization time-of-flight mass spectrometry for the study of Helicobacter pylori. Rapid Commun Mass Spectrom 2010;24:328-34. 
17. Barbuddhe SB, Maier T, Schwarz G, Kostrzewa M, Hof H, Domann E, et al. Rapid identification and typing of listeria species by matrix-assisted laser desorption ionization-time of flight mass spectrometry. Appl Environ Microbiol 2008;74:5402-7.

18. Mellmann A, Cloud J, Maier T, Keckevoet U, Ramminger I, Iwen $\mathrm{P}$, et al. Evaluation of matrix-assisted laser desorption ionization-time-of-flight mass spectrometry in comparison to 16S rRNA gene sequencing for species identification of nonfermenting bacteria. J Clin Microbiol 2008;46:1946-54.

19. Vanlaere E, Sergeant K, Dawyndt P, Kallow W, Erhard M, Sutton $\mathrm{H}$, et al. Matrix-assisted laser desorption ionisation-timeof-flight mass spectrometry of intact cells allows rapid identification of Burkholderia cepacia complex. J Microbiol Methods 2008;75:279-86.

20. Marklein G, Josten M, Klanke U, Muller E, Horre R, Maier T, et al. Matrix-assisted laser desorption ionization-time of flight mass spectrometry for fast and reliable identification of clinical yeast isolates. J Clin Microbiol 2009;47:2912-7.

21. Bizzini A, Durussel C, Bille J, Greub G, Prod'hom G. Performance of matrix-assisted laser desorption ionization-time of flight mass spectrometry for identification of bacterial strains routinely isolated in a clinical microbiology laboratory. J Clin Microbiol 2010;48:1549-54.

22. van Veen SQ, Claas EC, Kuijper EJ. High-throughput identification of bacteria and yeast by matrix-assisted laser desorption ionization-time of flight mass spectrometry in conventional medical microbiology laboratories. J Clin Microbiol 2010;48: 900-7.

23. Amiri-Eliasi B, Fenselau C. Characterization of protein biomarkers desorbed by MALDI from whole fungal cells. Anal Chem 2001;73:5228-31.

24. Erhard M, Hipler UC, Burmester A, Brakhage AA, Wostemeyer J. Identification of dermatophyte species causing onychomycosis and tinea pedis by MALDI-TOF mass spectrometry. Exp Dermatol 2008;17:356-61.

25. Hettick JM, Green BJ, Buskirk AD, Kashon ML, Slaven JE, Janotka E, et al. Discrimination of Aspergillus isolates at the species and strain level by matrix-assisted laser desorption/ionization time-of-flight mass spectrometry fingerprinting. Anal Biochem 2008;380:276-81.

26. Hettick JM, Green BJ, Buskirk AD, Kashon ML, Slaven JE, Janotka E, et al. Discrimination of Penicillium isolates by matrix-assisted laser desorption/ionization time-of-flight mass spectrometry fingerprinting. Rapid Commun Mass Spectrom 2008;22:2555-60.

27. Marinach-Patrice C, Lethuillier A, Marly A, Brossas JY, Gene J, Symoens $\mathrm{F}$, et al. Use of mass spectrometry to identify clinical Fusarium isolates. Clin Microbiol Infect 2009;15:634-42.

28. Santos C, Paterson RR, Venancio A, Lima N. Filamentous fungal characterizations by matrix-assisted laser desorption/ ionization time-of-flight mass spectrometry. J Appl Microbiol 2010;108:375-85.

29. La SB, Raoult D. Direct identification of bacteria in positive blood culture bottles by matrix-assisted laser desorption ionisation time-of-flight mass spectrometry. PLoS One 2009;4:e8041.
30. Christner M, Rohde H, Wolters M, Sobottka I, Wegscheider K, Aepfelbacher M. Rapid identification of bacteria from positive blood culture bottles by use of matrix-assisted laser desorptionionization time of flight mass spectrometry fingerprinting. J Clin Microbiol 2010;48:1584-91.

31. Ferroni A, Suarez S, Beretti JL, Dauphin B, Bille E, Meyer J, et al. Real-time identification of bacteria and Candida species in positive blood culture broths by matrix-assisted laser desorption ionization-time of flight mass spectrometry. J Clin Microbiol 2010;48:1542-8.

32. Stevenson LG, Drake SK, Murray PR. Rapid identification of bacteria in positive blood culture broths by matrix-assisted laser desorption ionization-time of flight mass spectrometry. J Clin Microbiol 2010;48:444-7.

33. Ferreira L, Sanchez-Juanes I, Porras-Guerra I, GarciaGarcia MI, Garcia-Sanchez JE, Gonzalez-Buitrago JM, et al. Microorganisms direct identification from blood culture by MALDI-TOF mass spectrometry. Clin Microbiol Infect 2011;17:546-51.

34. Prod'hom G, Bizzini A, Durussel C, Bille J, Greub G. Matrixassisted laser desorption ionization-time of flight mass spectrometry for direct bacterial identification from positive blood culture pellets. J Clin Microbiol 2010;48:1481-3.

35. Ferreira L, Sanchez-Juanes F, Gonzalez-Avila M, CembreroFucinos D, Herrero-Hernandez A, Gonzalez-Buitrago JM, et al. Direct identification of urinary tract pathogens from urine samples by matrix-assisted laser desorption ionization-time of flight mass spectrometry. J Clin Microbiol 2010;48:2110-5.

36. Cherkaoui A, Hibbs J, Emonet S, Tangomo M, Girard M, Francois $\mathrm{P}$, et al. Comparison of two matrix-assisted laser desorption ionization-time of flight mass spectrometry methods with conventional phenotypic identification for routine identification of bacteria to the species level. J Clin Microbiol 2010;48:1169-75.

37. Jackson KA, Edwards-Jones V, Sutton CW, Fox AJ. Optimisation of intact cell MALDI method for fingerprinting of methicillinresistant Staphylococcus aureus. J Microbiol Methods 2005;62:273-84.

38. Edwards-Jones V, Claydon MA, Evason DJ, Walker J, Fox AJ, Gordon DB. Rapid discrimination between methicillin-sensitive and methicillin-resistant Staphylococcus aureus by intact cell mass spectrometry. J Med Microbiol 2000;49:295-300.

39. Majcherczyk PA, McKenna T, Moreillon P, Vaudaux P. The discriminatory power of MALDI-TOF mass spectrometry to differentiate between isogenic teicoplanin-susceptible and teicoplanin-resistant strains of methicillin-resistant Staphylococcus aureus. FEMS Microbiol Lett 2006;255:233-9.

40. Bernardo K, Pakulat N, Macht M, Krut O, Seifert H, Fleer S, et al. Identification and discrimination of Staphylococcus aureus strains using matrix-assisted laser desorption/ionization-time of flight mass spectrometry. Proteomics 2002;2:747-53.

41. Du Z, Yang R, Guo Z, Song Y, Wang J. Identification of Staphylococcus aureus and determination of its methicillin resistance by matrix-assisted laser desorption/ionization timeof-flight mass spectrometry. Anal Chem 2002;74:5487-91. 